\title{
Affine Invariant Point-set Matching Using Convex Hull Bisection
}

\author{
Yue Li*, Bin Wang*, Yongsheng Gao*, Jun Zhou ${ }^{\dagger}$ \\ ${ }^{*}$ School of Engineering, Griffith University, Australia \\ ${ }^{\dagger}$ School of Information and Communication Technology, Griffith University, Australia \\ Emails: yue.li2@griffithuni.edu.au, \{bin.wang, yongsheng.gao,jun.zhou\}@griffith.edu.au
}

\begin{abstract}
Affine invariant point-set matching is an important issue in computer vision and pattern recognition. Using reference points derived from the convex hull of the point-set is an existing idea to solve this problem. However, how to choose proper and enough reference points for extracting affine invariant and powerful discriminative descriptors is an open problem. In this paper, a novel method termed convex hull bisection (CHB) is proposed for affine invariant point-set matching. In CHB, for each point in the point-set, two reference points are derived by bisecting the convex hull using the line connecting the point and the centroid of the convex hull. The resulting reference points and other reference points, such as the centroid point of the convex hull and the mean point of the point-set, are utilised to yield an 11-dimensional affine invariant feature vector associated with any point within the point-set. The obtained point based descriptors are used for point-set matching. The proposed method works well even in the case that reference points are overlapping or collinear, which overcomes the limitations of the existing convex hull based methods. The effectiveness of the proposed method is validated by an extensive experimental investigation.
\end{abstract}

\section{INTRODUCTION}

Point-set matching is an important topic in computer vision and pattern recognition. An object can be represented as a point-set, and the similarity of two objects can be determined by matching their corresponding point-sets. The key to the point-set matching is how to describe a point-set effectively. Zhang and Gao stated that for the object descriptor, the representative content and effectiveness were the significant representation issues [1]. The descriptors are expected to be affine invariant and discriminative, which are useful for the final goal of object recognition.

Many efforts have been made to find the affine invariant relationship between the elements of point-set for feature extraction. Liu et al. described that the point-set matching is always matching two point-sets with noise and outliers [2]. Most state-of-the-art image matching methods are based on frame and texture, which utilise sequential attributes as features. Cox and de Jager figured out that reducing the number of features in a large image is an efficient way for image matching [3]. Utilising feature points instead of lines is a way to reduce the number of features. Since both two-dimensional (2D) and three-dimensional (3D) images are constructed by points (pixels), an image can be treated as a point-set. Furthermore, an object matching problem is mainly to match object with its affine transformed model. Therefore point-set matching can be adapted to resolve image recognition and shape matching.
Researchers have developed a variety of point-set matching methods. Mikolajczyk and Schmid proposed an approach for detecting interest points with affine invariant property within an affine Gaussian scale-space [4]. Ho and Yang [5] treated points as complex numbers and interpreted that the corresponding objects were mutually transformed with a coefficient, which was computed from unit complex numbers without knowing the correspondence. Liu et al. [2] treated rigid point-set matching as the similarity of transformations on $\mathrm{x}$-axis and $\mathrm{y}$-axis, and took this similarity between point and its neighbours to calculate the matching similarity of point-sets. A rich descriptor was introduced by Grigorescu and Petkov [6] to describe spatial relationship between feature points. It contained the distances between given points to others. This descriptor was used to calculate the dissimilarity of feature points within point-set. Aiger and Kedem gave an asymptotically faster algorithm for rigid transformation and proposed novel algorithms for homothetic and similarity transformations [7]. McAuley and Caetano [8] specified a graphical model to solve the large point-set matching problem and adapted the model to handle occlusions. For non-rigid point-set matching, an algorithm was suggested to map nonrigid point-sets through using the joint estimation of thin-plate spline in [9]. Zia et al. [10] proposed a 3D point-set matching method based on hyperspectral images by using point-wise correspondence and they adapted it for the 3D reconstruction.

Convex hull was introduced to solve the point-set matching problem by Yang and Cohen [11]. They adapted invariance of area ratio for convex polygons to match vertices of convex hulls. Chen et al. utilised affine preserved feature points, named extended centroids, to constitute random triangles [12]. Extended centroids of an image were also extracted to reconstruct convex hull and build triangles [13]. Zhang et al. [14] applied extended centroid of a grayscale image and calculated affine invariant features using length ratio of parallel line segments. Fan et al. [15] introduced convex hull into 3D pointset matching. Gope and Kehtarnavaz [16] proposed a method to assemble triangles and extract barycentric coordinate as its invariant feature. An enhanced Hausdorff distance was suggested in their paper for measuring the similarity between point-sets. This method inspired our work, but it has some limitations on dealing with collinear and overlapping points.

In this paper, we propose a novel convex hull bisection method for point-set matching. In this method, extra reference 
points are derived by bisecting the convex hull associated with each point of the point-set. All available reference points are used to yield affine invariant features for describing the spatial relationship of the points. Some derived reference point independent features are also introduced for suppressing noise and enhancing the discriminative capability of shape matching method. An extensive experimental study is conducted to validate the effectiveness of the proposed method.

This paper is organised as follows. Section II reviews convex based point-set matching method and enhanced Hausdorff distance as well as their limitations. Section III describes the proposed affine invariant point-set matching method based on convex hull bisection. Then experimental results are presented in section IV. Finally, this paper is concluded in section V.

\section{Convex Based Point-Set Matching}

In this section, we will review convex hull based method with enhanced Hausdorff distance (CH-EHD) which inspired our work.

\section{A. Affine Invariants}

1) Convex Hull: The convex hull of a point-set is the area-minimising convex space, which contains all points. The properties of convex hull are given in [17]. An important property we can adapt to the point-set matching is that the convex hull is affine invariant [11], [18]. It means we can directly transform a convex hull instead of generating it from the transformed point-set.

2) Affine Preserved Points: Affine preserved point is a point that the notion of the point is always maintain under any affine transformation. Centroid is a well-known affine preserved point for any convex polygon [19]. For a convex polygon, the centroid point is always the centroid of polygon after affine transformation. The centroid of a convex hull is also affine preserved because convex hull in $2 \mathrm{D}$ space is a convex polygon [20]. Another applicable affine preserved point in point-set is the mean of points. Since the mean point of transformed point-set equals to transform mean point itself, it is affine preserved [16].

3) Barycentric Coordinate: Barycentric coordinate is the point coordinate lying in the plane of a triangle. For any point $p$, it can be formally written as follows:

$$
\begin{aligned}
& p=\alpha v_{1}+\beta v_{2}+\gamma v_{3} \\
& \text { s.t. } \quad \alpha+\beta+\gamma=1
\end{aligned}
$$

where $v_{1}, v_{2}, v_{3}$ are three vertices of a triangle and $[\alpha, \beta, \gamma]$ is the barycentric coordinate of $p$. Gope and Kehtarnavaz proved that the barycentric coordinate is affine invariant [16]. In [21], Floater et al. reviewed different barycentric coordinate generation methods and suggested that Wachspress coordinate [22] is positive and affine invariant in any convex polygon.

\section{B. Derived Reference Point}

For the generation of a triangle for any point in a pointset, a derived reference point is proposed by Gope and Kehtarnavaz [16]. This derived reference point is derived from

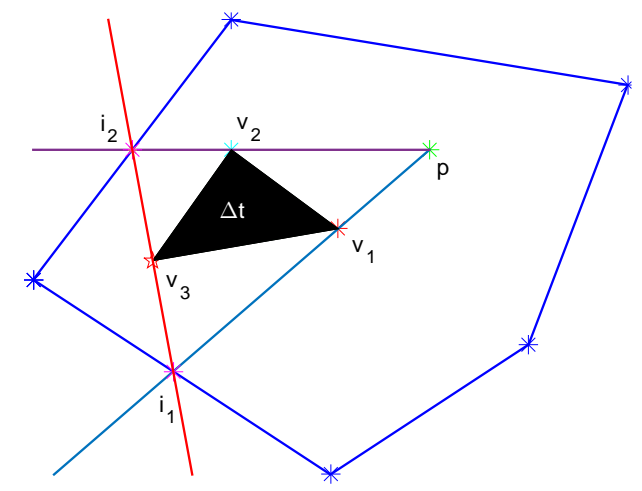

Fig. 1: Selection of reference points $v_{1}$ (the centroid), $v_{2}$ (the mean point), and derived reference point $v_{3}$ for feature point p. $\Delta t$ is the triangle constructed by vertices $v_{1}, v_{2}$ and $v_{3}$.

other two affine persevered points and it is related to the shape of the convex hull. Figure 1 shows how to calculate the derived reference point $v_{3}$. Given a point-set $S=\left\{s_{1}, s_{2}, \ldots, s_{m}\right\}$ and its convex hull $C H=\left\{c h_{1}, c h_{2}, \ldots, c h_{n}\right\} . v_{1}$ is the centroid of $C H, v_{2}$ denotes the mean point of $S$. For a random point $p(p \in S)$, draw two rays: $\overrightarrow{p v_{1}}$ and $\overrightarrow{p v_{2}}$, the intersections of rays to the edges of convex hull are $i_{1}$ and $i_{2}$. Connecting $i_{1}$ and $i_{2}$ by a line, let $v_{3}$ be the midpoint of line segment $i_{1} i_{2}$. It can be proved that $v_{3}$ is affine preserved since:

1) the intersection of two lines is preserved under affine transformation, and

2) the midpoint of a line segment is affine invariant.

Therefore, a triangle $\Delta t$ is generated using these three affine preserved vertices $\left[v_{1}, v_{2}, v_{3}\right]$. Then the barycentric coordinate of $p$ to $\Delta t$ can be calculated.

\section{Enhanced Hausdorff Distance}

In [23], Huttenlocher et al. applied Hausdorff distance to point-set matching. Gao and Leung extended it to measure the distance between line segments [24]. Dubuisson and Jain utilised a modified Hausdorff distance (MHD) as the mean distance of two sets [25]. Given two point-sets $U=\left\{u_{1}, u_{2}, \ldots, u_{j}\right\}$ and $V=\left\{v_{1}, v_{2}, \ldots, v_{k}\right\}$, the modified Hausdorff distance is defined as:

$$
H(U, V)=\max (h(U, V), h(V, U))
$$

where $h(U, V)$ is a directive distance from $U$ to $V$ :

$$
h(U, V)=\frac{1}{j} \sum_{u \in U} d(u, V)
$$

The $d(u, V)$ represents the minimum distance from point $u$ to point-set $V$, which is defined as:

$$
d(u, V)=\min _{v \in V}\|u-v\|
$$

Here, $\|\cdot\|$ is a norm.

The enhanced Hausdorff distance (EHD) applies the MHD and adapts the number of competitive points with a weight. 


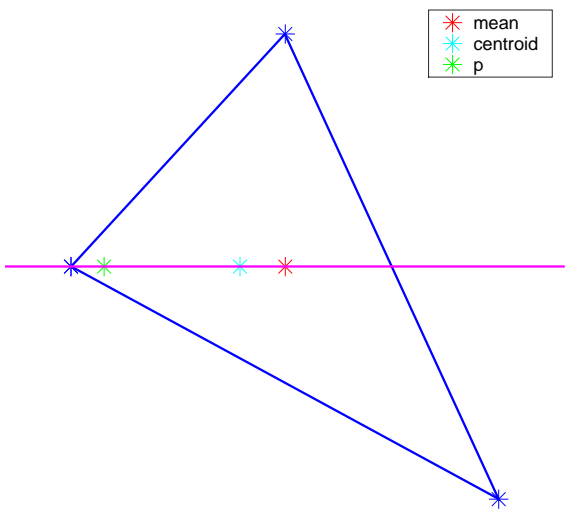

(a)

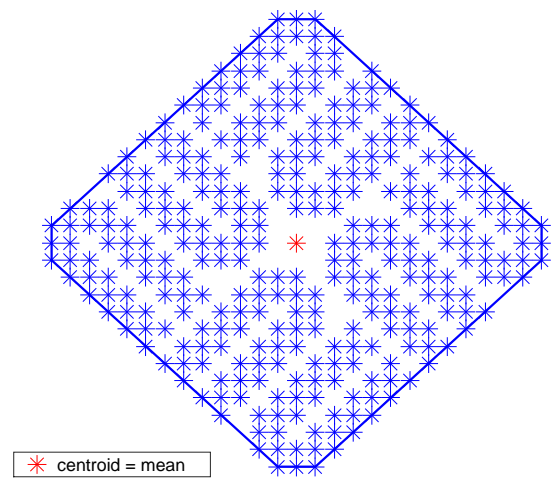

(b)

Fig. 2: Two samples of point-set under limitations: (a) point $p$ is collinear with the mean point and the centroid; (b) the mean point and the centroid are overlapping.

By modifying Equation (3), the directive distance $h_{e}(U, V)$ is calculated as:

$$
h_{e}(U, V)=\frac{1}{j-\psi} \sum_{u \in U} d(u, V)
$$

where $d(u, V)$ is the same as in Equation (4). If $l$ points $(l>1)$ in $U$ matches to same point $v \in V$, there are $l-1$ competitive points for $v$. The weight $\psi$ is calculated by summing all competitive points for the set $V$. The calculation of $\psi$ follows these steps:

1) Generate a $k$-dimensional array $\Lambda$ with all cells initialised by -1 ;

2) Find the nearest point in $V$ for each $u \in U$, and increase the corresponding cell in $\Lambda$ by 1 ;

3) $\psi$ equals to the sum of all cells with value greater than 0 in $\Lambda$.

Since $\psi$ contains the number of competitive points, $j-\psi$ removes all competition points so that EHD increases the discriminative power of matching when competitive points exist.

\section{Limitations}

The method proposed by Gope and Kehtarnavaz uses the centroid of the convex hull and mean point of point-set to calculate a derived reference point, and then generates a triangle with these three points [16]. However, in some situations, this method is insufficient to derive a proper reference point. Firstly, as shown in Figure 2(a), when a point $(p)$ is collinear with the centroid and the mean point, finding the derived reference point for point $p$ is impossible. Thus, a triangle cannot be built for gathering barycentric coordinate. Secondly, for any centrosymmetric point-set, i.e., the case shown in Figure 2(b), the reference points are overlapping. Any point within the point-set cannot generate a derived reference point and construct a feature. The third limitation is that the derived reference point is highly dependent on the edge of the convex hull. When the shape of the convex hull is largely impacted by noises, the derived reference point varies significantly, so the performance of matching is not stable under noises.

\section{Matching Using Convex Hull Bisection}

In this section, we introduce the convex hull bisection (CHB) method to divide convex hull into two convex polygons and apply affine preserved points of these polygons to extract affine invariant features. We utilise these features to generate an affine invariant descriptor of points.

\section{A. Affine Transformation and Invariance}

An affine transformation is an important geometric transformation adopted by computer vision. It includes translation, rotation, skewing and scaling operations. An affine transformation is also a subgroup of the projective transformation. Mathematically, an affine transformation of 2D object can be defined as follows. Given a set of points $S$, the transformation equation is:

$$
S^{\prime}=A S+B
$$

where $S^{\prime}$ is the transformed set, $A$ is a 2-by-2 transformation matrix and $B$ represents a translation vector. For any point with coordinate $[x, y]$ in $S$, the transformation can be written as:

$$
\left[\begin{array}{l}
x^{\prime} \\
y^{\prime}
\end{array}\right]=\left[\begin{array}{ll}
a_{11} & a_{12} \\
a_{21} & a_{22}
\end{array}\right]\left[\begin{array}{l}
x \\
y
\end{array}\right]+\left[\begin{array}{l}
b_{1} \\
b_{2}
\end{array}\right]
$$

The areas of the transformed 2D objects are scaled by $\operatorname{det}|A|$, where $\operatorname{det}|\cdot|$ denotes the matrix determinant. Thus, the area ratio of graphs is invariant to the same affine transformation. So we can apply area ratio as the feature to construct our descriptor.

\section{B. Convex Hull Bisection}

For any point-set $S=\left\{s_{1}, s_{2}, \ldots, s_{m}\right\}$, let its convex hull be $C H=\left\{c h_{1}, c h_{2}, \ldots, c h_{n}\right\}(n \leq m)$ and the centroid be $c$. For any point $p \neq c$ in $S$, we can draw a line $p c$. The intersections of $p c$ and the convex hull $C H$ are $i_{1}$ and $i_{2}$. Then the convex hull is divided into two convex polygons 


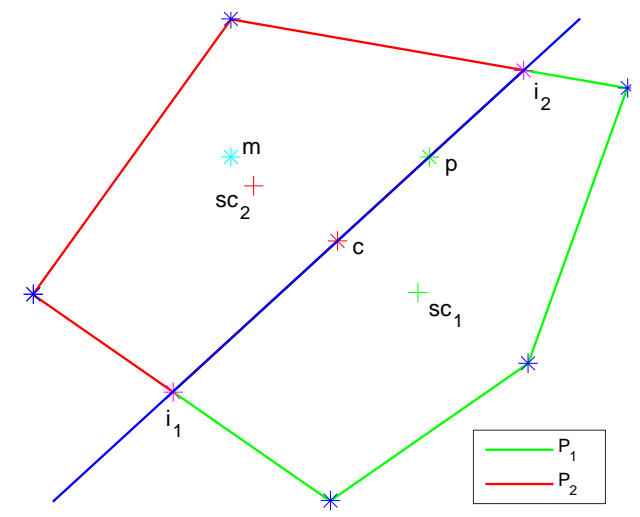

Fig. 3: Convex hull bisection into two convex polygons $\left(P_{1}\right.$ and $\left.P_{2}\right)$ for feature point $p$, and the affine preserved points $(c$, $m, s c_{1}$ and $s c_{2}$ ).

$P_{1}=\left\{c h_{1}, \ldots, c h_{k}, i_{1}, i_{2}\right\}$ and $P_{2}=\left\{c h_{k+1}, \ldots, c h_{n}, i_{1}, i_{2}\right\}$. We name it as the Convex Hull Bisection associated with the given point $p$. Let $s c_{1}$ and $s c_{2}$ denote the centroids of the convex polygon $P_{1}$ and the convex polygon $P_{2}$ respectively. Figure 3 gives an example to illustrate the proposed convex hull bisection.

The intersections $i_{1}$ and $i_{2}$ are preserved because the intersection of two straight lines is preserved under affine transformation. Furthermore, transformed polygons $P_{1}^{\prime}$ and $P_{2}^{\prime}$ maintain the vertices points in the transformed convex hull $C H^{\prime}=\left\{c h_{1}^{\prime}, c h_{2}^{\prime}, \ldots, c h_{n}^{\prime}\right\}$ because points in $C H$ are transformed under the same transformation. All points on the transformed straight line $p^{\prime} c^{\prime}$ can be calculated by transforming each point on straight line $p c$ separately. So transformed intersections $i_{1}^{\prime}$ and $i_{2}^{\prime}$ are the same as transforming $i_{1}$ and $i_{2}$ directly. Therefore, all points in $P_{1}^{\prime}=\left\{c h_{1}^{\prime}, \ldots, c h_{k}^{\prime}, i_{1}^{\prime}, i_{2}^{\prime}\right\}$ and $P_{2}^{\prime}=\left\{c h_{k+1}^{\prime}, \ldots, c h_{n}^{\prime}, i_{1}^{\prime}, i_{2}^{\prime}\right\}$ are affine preserved. The bisected convex hulls are affine invariant. It is easy to prove that the centroids of $P_{1}^{\prime}$ and $P_{2}^{\prime}$ are the same as transforming $s c_{1}$ and $s c_{2}$. We can apply the centroids as the reference points to construct features. From now on, the reference points are defined as $R=\{c, m, s c 1, s c 2\}$.

Centroids of bisected convex hulls can be seen as a kind of extended centroids. Since all extended centroids and centroid are linearly distributed (which is described in [13]), $c, s c_{1}$ and $s c_{2}$ are always collinear. Therefore, it is important that we shall never use these three points as vertices to construct a triangle. Although we have these four affine preserved feature points, they are not enough to represent the point $p$ in the matching problem. We still need to construct an affine invariant descriptor for $p$.

\section{Affine Invariant Feature Descriptor}

As mentioned above, the area ratio of objects is affine invariant. To extract feature descriptor, we adapt area ratio of triangles, which is constructed by the reference points. To remove the limitation of overlapping reference points, we calculate the area of triangles by two reference points $\left(v_{1}\right.$ and $v_{2}$ ) for any point $p$ in a point-set $S=\left\{s_{1}, s_{2}, \ldots, s_{n}\right\}$ as follows:

$$
A_{p}(v 1, v 2)= \begin{cases}\operatorname{area}\left(\Delta\left(v_{1}, v_{2}, v_{3}\right)\right) & \text { if } v_{1} \neq v_{2} \\ 0 & \text { otherwise }\end{cases}
$$

where $v_{1} \in R, v_{2} \in R$, and $v_{3}$ is the derived reference points using the method in section II-A3) by $v_{1}, v_{2}$ and $p$. The area $(\cdot)$ denotes the function to calculate the triangular area with three vertices.

We use six groups of reference points as $v_{1}$ and $v_{2}$ respectively: 1) $c$ and $\left.s c_{1}, 2\right) c$ and $\left.s c_{2}, 3\right) m$ and $\left.s c_{1}, 4\right) m$ and $\left.s c_{2}, 5\right) s c_{1}$ and $\left.s c_{2}, 6\right) c$ and $m$, where $m$ is the mean point of point-set, $c$ is the centroid, $s c_{1}$ and $s c_{2}$ are the centroids of bisected convex hulls. Among these six groups, except the worst case that $p$ and $c$ are overlapping, at least the first five groups are non-zero. It should be noted that if $c$ and $m$ are overlapping, $A_{p}(c, m)=0, A_{p}\left(c, s c_{1}\right)=A_{p}\left(m, s c_{1}\right)$ and $A_{p}\left(c, s c_{2}\right)=A_{p}\left(m, s c_{2}\right)$.

The areas of triangles are not stable under noises. This is because the triangles are constructed using the derived reference points which are calculated based on the intersections of straight lines and the edges of the convex hull. The shape of the convex hull is heavily affected by noises. We say these features are derived reference point dependent (DRPDependent). To reduce the impact of noises, we define another 5 derived reference point independent (DRP-Independent) features: 1) area $(\Delta(m, c, p))$, 2) $\left.A_{m}^{\prime}(p, m), 3\right) A_{m}^{\prime}(p, c)$, 4) $A_{s}^{\prime}(p, m)$, 5) $A_{s}^{\prime}(p, c)$, where $A_{m}^{\prime}(\cdot)$ is the mean area of all triangles, which is defined as:

$$
A_{m}^{\prime}(p, c)=\frac{1}{n-1} \sum_{i=1, s_{i} \neq p}^{n} \operatorname{area}\left(\Delta\left(p, c, s_{i}\right)\right)
$$

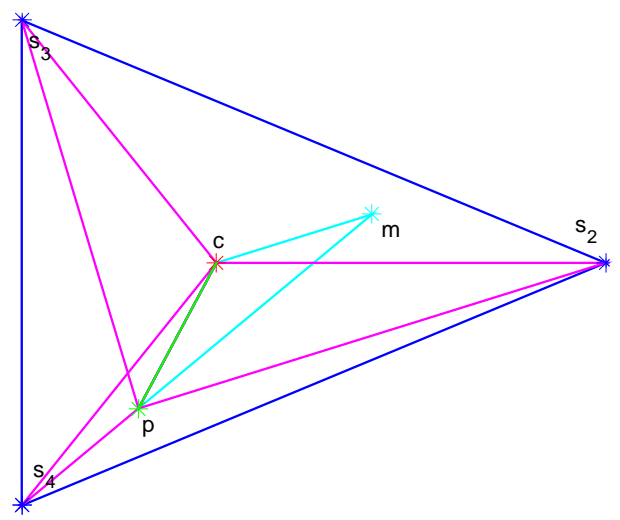

Fig. 4: DRP-Independent triangles for feature point $p=s_{1}$ $\left(s_{1} \in S\right)$. The cyan triangle is the $\Delta(m, c, p)$. The pink triangles are the triangles used in $A_{m}^{\prime}(p, c)$ and $A_{s}^{\prime}(p, c)$. The green line segment is the overlapping edge of $\Delta(m, c, p)$ and $\Delta\left(p, c, s_{3}\right)$. 
The standard division of triangle areas $A_{s}^{\prime}(\cdot)$ is:

$$
A_{s}^{\prime}(p, c)=\sqrt{\frac{1}{n-2} \sum_{i=1, s_{i} \neq p}^{n}\left(\operatorname{area}\left(\Delta\left(p, c, s_{i}\right)\right)-A_{m}^{\prime}(p, c)\right)^{2}}
$$

These two features are calculated by summing all triangle areas together. These triangles are constructed by the points in point-set and the corresponding reference points. Figure 4 gives an illustration of DRP-Independent triangles. For the situation that $m$ and $c$ are overlapping, the DRP-Independent features have area $(\Delta(m, c, p))=0, A_{m}^{\prime}(p, c)=A_{m}^{\prime}(p, m)$ and $A_{s}^{\prime}(p, c)=A_{s}^{\prime}(p, m)$. If $p=m$, all features constructed using point $p$ and reference point $m$ are equal to 0 .

Now we can define our descriptor for point $p$ as:

$$
\vec{f}(p)= \begin{cases}N a N & \text { if collinear }(p, c, m) \text { or } p=c \\ \overrightarrow{f^{\prime}}(p) & \text { otherwise }\end{cases}
$$

where collinear $(\cdot)$ is a boolean function, which is used to test if $p$ is collinear with any other two reference points. $\overrightarrow{f^{\prime}}(\cdot)$ is formally defined as follows:

$$
\begin{array}{r}
\overrightarrow{f^{\prime}}(p)=\left[\left(A_{p}(c, m), A_{p}\left(c, s c_{1}\right), A_{p}\left(c, s c_{2}\right), A_{p}\left(m, s c_{1}\right),\right.\right. \\
A_{p}\left(m, s c_{2}\right), A_{p}\left(s c_{1}, s c_{2}\right), \operatorname{area}(\Delta(m, c, p)), \\
\left.\left.A_{m}^{\prime}(p, m), A_{m}^{\prime}(p, c), A_{s}^{\prime}(p, m), A_{s}^{\prime}(p, c)\right)\right]
\end{array}
$$

Since collinear reference points are always collinear under affine transformation, we can ignore them when constructing features. Regarding the particular situation when $p$ and $c$ are overlapping, we treat it as the same as they are collinear. It means that the vector of $p$ will be directly ignored when constructing features, because this point preserves overlapping under transformation. The descriptor for any point in a centrosymmetric point-set has two areas equal to 0 , i.e. $A_{p}(c, m)$, $\operatorname{area}(\Delta(m, c, p))$, and four pairs with the same areas except $A_{p}\left(s c_{1}, s c_{2}\right)$. Using all points in set $S$, we can construct the descriptor matrix as $f_{s}=\left\{\vec{f}\left(s_{1}\right), \vec{f}\left(s_{2}\right), \ldots, \vec{f}\left(s_{n}\right)\right\}$. This matrix is normalised by the maximum value of each column in the descriptor.

Now, we have defined a normalised descriptor as the affine invariant feature descriptor to represent point $p$, and it addressees the limitation on reference points collinear and overlapping situations. Then we can apply this descriptor to match the points in other point-sets by calculating the distance between features.

\section{Matching Process}

When matching two point-sets $S_{X}=\left\{s_{x 1}, s_{x 2}, \ldots, s_{x j}\right\}$ and $S_{Y}=\left\{s_{y 1}, s_{y 2}, \ldots, s_{y k}\right\}$, a feature vector is generated for each point in both sets. We use these vectors to construct two descriptor matrices $f_{X}=\left\{\vec{f}\left(s_{x 1}\right), \vec{f}\left(s_{x 2}\right), \ldots, \vec{f}\left(s_{x j}\right)\right\}$ and $f_{Y}=\left\{\vec{f}\left(s_{y 1}\right), \vec{f}\left(s_{y 2}\right), \ldots, \vec{f}\left(s_{y k}\right)\right\}$. Then we apply enhanced Hausdorff distance to achieve higher discriminative capability as follows:

$$
H_{e}\left(f_{X}, f_{Y}\right)=\max \left(h_{e}\left(f_{X}, f_{Y}\right), h_{e}\left(f_{Y}, f_{X}\right)\right)
$$

where $h_{e}$ is calculated using Equation (5). The $L^{2}$-norm is used to calculate the minimum distance from a point in $S_{X}$ to the point in $S_{Y}$ via Equation (4). The distance between points is formally written as:

$$
d_{e}\left(f_{x}, f_{Y}\right)=\min _{f_{y} \in f_{Y}}\left\|f_{x}-f_{y}\right\|_{2}
$$

$H_{e}$ is the distance of point-sets $S_{X}$ and $S_{Y}$. The smaller this distance is, the more similar two point-sets are.

\section{EXPERIMENTS}

We have described the proposed CHB method in the previous section. Now we show its performance on resolving the point-set matching problem, and its discriminative capability.

\section{A. Experiments on Point-set Matching with Limitations}

To study how our method deals with collinear limitation, we generate four point-sets including one containing collinear points as in Figure 5. We compare each set to others by using $\mathrm{CH}-\mathrm{EHD}$ and the proposed method. The produced distance matrix is shown in Table I. The matrix includes distances from every set to the others, which is calculated by EHD. The distances here are not significant to show the capability of matching performance of two methods, which will be discussed further in the following experiments. This experiment is only to demonstrate that how these methods perform under certain situations. It can be observed that the CH-EHD fails when comparing collinear set $d$ with others, but our method works properly.

We use the same method as in [2], [26] to generate the point overlapping dataset. We generate twenty-five point-sets with 70 points in each set. These sets contain 5 centrosymmetric point-sets to simulate the point overlapping situation. Three arbitrary transformed sets are generated for each set. Each set and its transformed sets are classified into one class. Figure 6

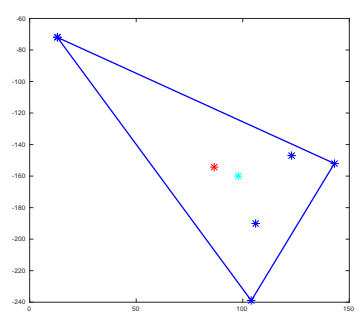

(a)

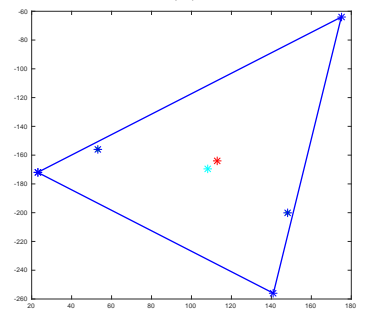

(c)

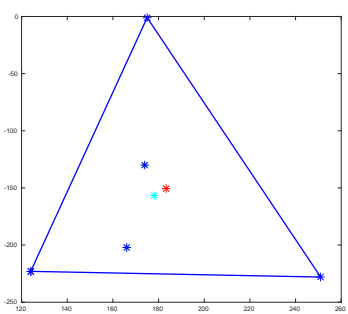

(b)

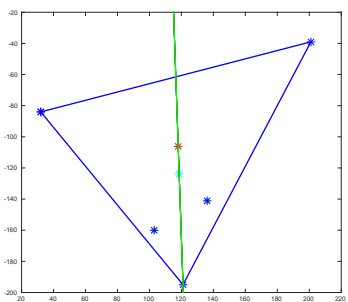

(d)
Fig. 5: Point-sets for experiments: (a)-(c) normal point-sets; (d) point-set with collinear points. 
TABLE I: Distance Matrix for Collinear Sets

\begin{tabular}{|c|c|c|c|c|c|c|c|c|}
\hline & \multicolumn{4}{|c|}{ CH-EHD [16] } & \multicolumn{4}{c|}{ Proposed CHB } \\
\hline & $\mathrm{a}$ & $\mathrm{b}$ & $\mathrm{c}$ & $\mathrm{d}$ & $\mathrm{a}$ & $\mathrm{b}$ & $\mathrm{c}$ & $\mathrm{d}$ \\
\hline $\mathrm{a}$ & 0 & 0.28 & 0.33 & Fail & 0 & 0.99 & 0.48 & 0.72 \\
\hline $\mathrm{b}$ & 0.28 & 0 & 0.34 & Fail & 0.99 & 0 & 0.97 & 1.69 \\
\hline $\mathrm{c}$ & 0.33 & 0.34 & 0 & Fail & 0.48 & 0.97 & 0 & 1.06 \\
\hline $\mathrm{d}$ & Fail & Fail & Fail & Fail & 0.72 & 1.69 & 1.06 & 0 \\
\hline
\end{tabular}

illustrates the synthetic dataset. In the experiment, we apply EHD to calculate the distances from one set (the probe set) to all other sets. If the nearest set and the probe set are in the same class, it is a correct matching. Otherwise, it is an incorrect matching. We also add zero-mean $n$ variance Gaussian noise to all point-sets to check the robustness of different methods to noises. The results are shown in Figure 7.

It can be obviously found that our method works favourably at the position of 0 variances. But $\mathrm{CH}$-EHD fails at that position. When noise is added, point-sets are not overlapping anymore, so the correct matching ratio increases for CH-EHD at 1 variance. The results also show that our method is stable than $\mathrm{CH}-\mathrm{EHD}$ under noise. Because they are highly related

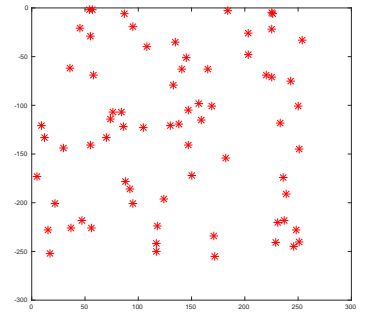

(a)

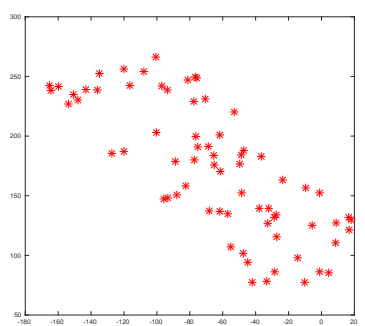

(c)

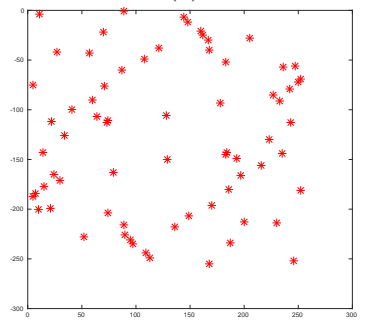

(e)

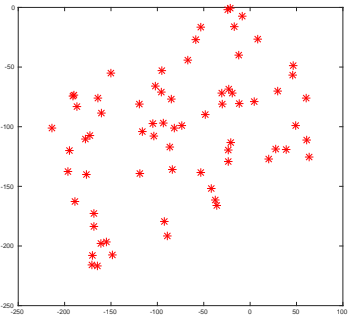

(b)

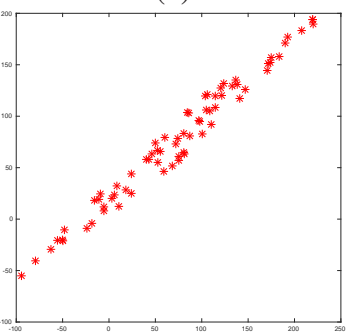

(d)

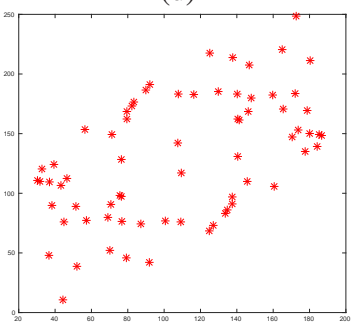

(f)
Fig. 6: Samples of synthetic dataset: (a) the original pointset without centrosymmetric; (b)-(d) three arbitrary transformed point-sets of (a); (e) the original point-set with centrosymmetric; (f) arbitrary transformed point-set of (e).

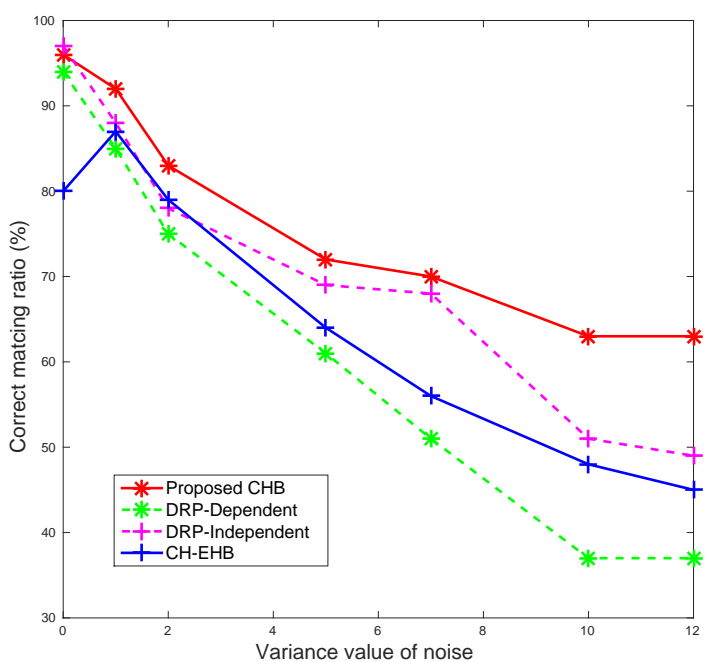

Fig. 7: Experiment results of point-set matching under overlapping limitation and noise.

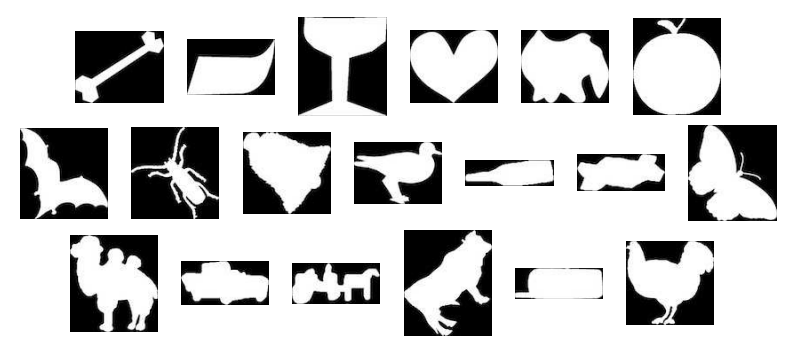

Fig. 8: Samples images from MPEG-7 CE-Shape-1 dataset.

to the derived reference points, the performance of CHB and DRP-Dependent only drops dramatically with large values of the variance of Gaussian noise. With DRP-Independent only, our method performs at a better level on stability than $\mathrm{CH}-$ EHD and DRP-Dependent only.

\section{B. Experiments on Shape Matching}

We use three image datasets to evaluate the shape matching performance of different methods. Instead of wrapped images, we are interested in evaluating our method with a higher intra-shape-variant dataset. The first dataset is MPEG-7 Core Experiment CE-Shape-1 (MPEG-7 CE-Shape-1) dataset [27] as shown in Figure 8. As Wang and Gao suggested this database was widely used to estimate the performance of shape recognition methods [28]. This dataset contains 70 different classes. Each class has 20 binary shape images. The images within each class have highly intra-shape-variance (Figure 9). Jiang et al. applied four rigid transformations to each object [29]. We directly test our proposed method using all images from dataset without any transformation operation. We manually apply Canny edge detection method [30] to extract edges of images and treat the edge map as a pointset. Ten images are randomly sampled from each class in the dataset. Then we have $10 \times 70=700$ images in the gallery, and the rest 700 images are probes. 


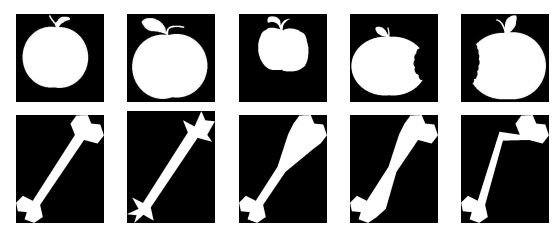

Fig. 9: Sample of intra-shape-variant images within MEPG-7 CE-Shape-1 dataset.

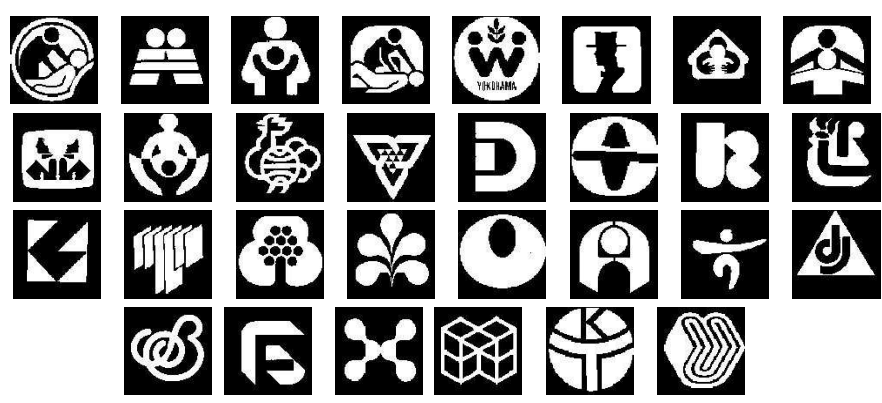

Fig. 10: Original images within transformed classes from MPEG-7 CE-2 A4 dataset.

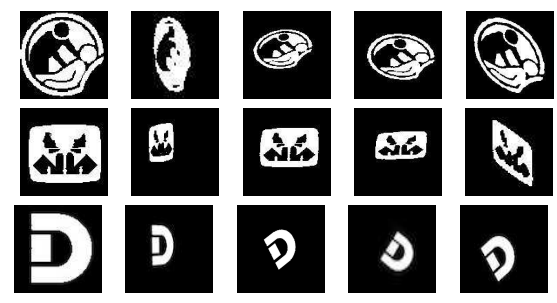

Fig. 11: Sample images from labeled classes, images in first column are original images, others are transformed image from original images.

The second dataset is MPEG-7 region shape dataset CE-2 A4 set (MPEG-7 CE-2 A4). This dataset contains 3101 binary images. Among them, 330 images are labelled as transformed images in this dataset. These 330 images are classified into 30 classes with 11 images in each class. There is 1 original (as shown in Figure 10) image in each class and the rest 10 images in class are transformed from the original image. Sample transformed images in this dataset are shown in Figure 11. We use these 330 transformed images as a dataset in our experiment. Five random images from each class are used as probes and the others construct a gallery.

Because the existing public available datasets do not fulfil our experiment requirement, i.e., images are with affine transformation only, we also create a simulated dataset. In this dataset, we manually collect 36 vehicle logo images from the Internet. There are no separate parts in all logos, which means that all logos can be represented as a closed contour object. For each logo, we create 9 arbitrary affine transformations using Equation (6). In total, we have 360 images in 36 classes. We apply the same testing protocol as MPEG-7 CE-Shape1 dataset, using 180 random probes (5 in each class) and 180 gallery images. Sample images of this dataset are given in Figure 12. Figure 13 shows arbitrary affine transformed images.

Because the purpose of this experiment is to show how our point-set matching method works for image matching, we design an experiment for seeking the most similar image in the gallery of a probe to verify if the probe is an affine transformed sample of the gallery image. Each probe is used to match with images within the gallery to check if the probe and its nearest image are in the same class. If they are in the same class, there is a correct match. The correct matching ratio was calculated by:

$$
\text { Ratio }=\frac{\text { Number of Correct Matchings }}{\text { Number of Probes }} \times 100 \%
$$

To prove that our method maintains recognition capability while keeping the affine invariant property, we adopt Inner-distance Shape Context with Dynamic Programming (IDSC+DP) method [31] and Height Functions (HF) method [32] as benchmarks for the matching experiment. The results are shown in Table II. Performance for DRP-Dependent and DRP-Independent features only are given as well.

TABLE II: Results of Shape Matching Experiments

\begin{tabular}{|l||c|c|c|}
\hline Method & $\begin{array}{c}\text { MPEG-7 } \\
\text { CE-Shape-1 }\end{array}$ & $\begin{array}{c}\text { MPEG-7 } \\
\text { CE-2 A4 }\end{array}$ & $\begin{array}{c}\text { Simulated } \\
\text { Set }\end{array}$ \\
\hline Proposed CHB & $87.14 \%$ & $\mathbf{9 5 . 3 3 \%}$ & $\mathbf{8 2 . 2 2 \%}$ \\
\hline DRP-Dependent features & $71.86 \%$ & $91.33 \%$ & $74.44 \%$ \\
\hline DRP-Independent features & $76.71 \%$ & $90.00 \%$ & $71.67 \%$ \\
\hline CH-EHD [16] & $65.28 \%$ & $89.33 \%$ & $61.67 \%$ \\
\hline IDSC+DP [31] & $\mathbf{9 2 . 0 0 \%}$ & - & $41.67 \%$ \\
\hline HF [32] & $85.42 \%$ & - & $55.56 \%$ \\
\hline
\end{tabular}

In the first column of Table II, our CHB method demonstrates competitive matching capability comparing with image-

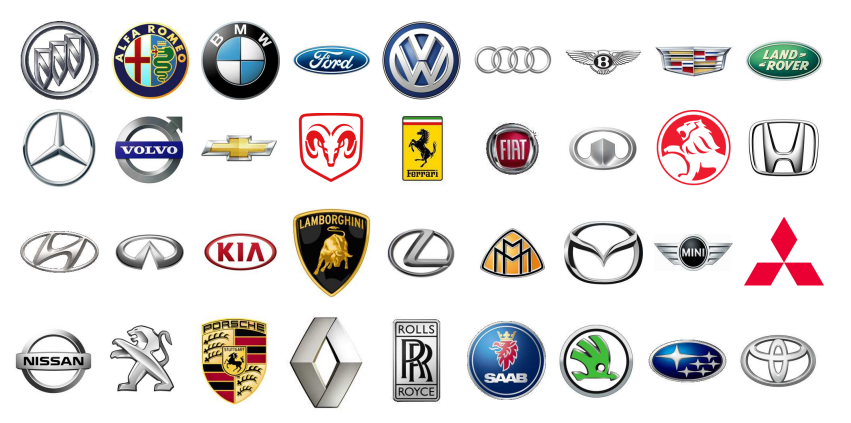

Fig. 12: Sample logo images of simulated dataset.

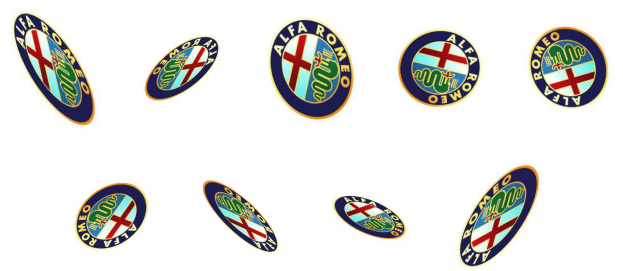

Fig. 13: Samples of arbitrary affine transformed logo. 
matching-specific approaches IDSC+DP and HF. Because the MPEG-7 CE-Shape-1 dataset is not affine-wrapped, CH-EHD does not produce good results. In the second column of the table, it can be seen that the proposed CHB method has the highest correct matching ratio on MPEG-7 CE-2 A4 dataset. IDSC+DP and $\mathrm{CH}$ methods are unable to deal with this dataset because some images contain separate parts (as shown in the third row of Figure 11). According to the last column of the table, the proposed CHB method demonstrates the best performance among all methods. Comparing with affine invariant methods, IDSC+DP and HF methods indicate their limitations on the affine-wrapped dataset. Among experiments on all three datasets, our method with DRP-Dependent and DRP-Independent achieve higher performance than CH-EHD.

\section{CONCLUSION}

In this paper, we have introduced an affine invariant pointset matching approach. This approach adapts convex hull bisection for each point in point-set, to derive affine preserved points as reference points. The corresponding reference points are used to construct a feature descriptor for each point to represent the spatial information of point-set. Our approach addresses the limitations in overlapping and collinear situation. The experimental results have validated its effectiveness. It is also proved by the experiments that, comparing with other benchmark methods, our approach has higher discriminative power while maintaining the affine invariant property. The image matching performance for our method can be further boosted by applying feature points instead of edge points extracted by Canny edge detector. Although our method is partially robust to noise, it can be improved by developing more DRP-Independent features and removing all DRP-Dependent features.

\section{ACKNOWLEDGMENT}

This work was supported in part by the Australian Research Council under Grant DP140101075 and in part by the National Natural Science Foundation of China under Grant 61372158.

\section{REFERENCES}

[1] B. Zhang, Y. Gao, S. Zhao, and J. Liu, "Local derivative pattern versus local binary pattern: Face recognition with high-order local pattern descriptor," IEEE Transactions on Image Processing, vol. 19, no. 2, pp. 533-544, 2010.

[2] X. Liu, C. Han, and T. Guo, "A Robust Point Sets Matching Method," in Advances in Swarm and Computational Intelligence, 2015, pp. 383-390.

[3] G. Cox and G. de Jager, "A Survey of Point Pattern Matching Techniques and A New Approach to Point Pattern Recognition," in Proceedings of the 1992 South African Symposium on Communications and Signal Processing. IEEE, 1993, pp. 243-248.

[4] K. Mikolajczyk and C. Schmid, "An Affine Invariant Interest Point Detector," in 7th European Conference on Computer Vision, 2002, pp. $128-142$.

[5] J. Ho and M.-H. Yang, "On affine Registration of Planar Point Sets Using Complex Numbers," Computer Vision and Image Understanding, vol. 115 , no. 1 , pp. 50-58, 2011.

[6] C. Grigorescu and N. Petkov, "Distance Sets for Shape Filters and Shape Recognition," IEEE Transactions on Image Processing, vol. 12, no. 10 , pp. 1274-1286, 2003.

[7] D. Aiger and K. Kedem, "Approximate Input Sensitive Algorithms for Point Pattern Matching," Pattern Recognition, vol. 43, no. 1, pp. 153-159, 2010.
[8] J. J. McAuley and T. S. Caetano, "Fast Matching of Large Point Sets Under Occlusions," Pattern Recognition, vol. 45, no. 1, pp. 563-569, 2012.

[9] H. Chui and A. Rangarajan, "A New Point Matching Algorithm for Non-rigid Registration," Computer Vision and Image Understanding, vol. 89, no. 2-3, pp. 114-141, 2003.

[10] A. Zia, J. Liang, J. Zhou, and Y. Gao, "3D Reconstruction from Hyperspectral Images," 2015 IEEE Winter Conference on Applications of Computer Vision (WACV), pp. 318-325, 2015.

[11] Z. Yang and F. Cohen, "Image Registration and Object Recognition Using Affine Invariants and Convex Hulls," IEEE Transactions on Image Processing, vol. 8, no. 7, pp. 934-946, 1999.

[12] T. Chen, Y. Su, Y.-M. Jiang, T. Tnag, and W.-X. Yu, "Affine Invariant Feature Extraction Based on Affine Geometry," Journal of Image and Graphics, vol. 12, no. 9, pp. 1633-1641, 2007.

[13] L. Lei, T. Chen, Z.-Y. Li, and Y. Su, "A New Approach to Image Invariant Extraction Under Global Affine Transformation," Journal of National University of Defense Technology, vol. 4, pp. 64-70, 2008.

[14] Y. Zhang, K. Lu, and Y.-H. Gao, "Fast Image Matching Algorithm Based on Affine Invariants," Journal of Central South University, vol. 21, no. 5, pp. 1907-1918, 2014.

[15] J. Fan, J. Yang, F. Lu, D. Ai, Y. Zhao, and Y. Wang, "3-Points Convex Hull Matching (3PCHM) for Fast and Robust Point Set Registration," Neurocomputing, pp. 1-14, 2016.

[16] C. Gope and N. Kehtarnavaz, "Affine Invariant Comparison of Point-sets Using Convex Hulls and Hausdorff Distances," Pattern Recognition, vol. 40, no. 1, pp. 309-320, 2007.

[17] M. Preparata, Franco P. Shamos, Computational Geometry: An Introduction. Springer Science \& Business Media, 2012.

[18] J. Flusser, "Affine Invariants of Convex Polygons," IEEE Transactions on Image Processing, vol. 11, no. 9, pp. 1117-1118, 2002.

[19] M. Meyer, C. Schuett, and E. M. Werner, "Affine Invariant Points," arXiv, pp. 1-24, 2013.

[20] B. Grünbaum, "Measures of Symmetry for Convex Sets," Proceedings of Symposia in Pure Mathematics, vol. 7, pp. 233-270, 1963.

[21] M. S. Floater, K. Hormann, and G. Kós, "A General Construction of barycentric Coordinates Over Convex Polygons," Advances in Computational Mathematics, vol. 24, no. 1-4, pp. 311-331, 2006.

[22] E. L. Wachspress, A Rational Finite Element Basis. New York: Academic Press, 1975.

[23] D. Huttenlocher, G. Klanderman, and W. Rucklidge, "Comparing Images Using the Hausdorff Distance," IEEE Transactions on Pattern Analysis and Machine Intelligence, vol. 15, no. 9, pp. 850-863, 1993.

[24] Y. Gao and M. K. H. Leung, "Face recognition using line edge map," IEEE Transactions on Pattern Analysis and Machine Intelligence, vol. 24, no. 6, pp. 764-779, 2002.

[25] M.-P. Dubuisson and A. Jain, "A Modified Hausdorff Distance For Object Matching," in Proceedings of 12th International Conference on Pattern Recognition, vol. 1, no. 1. IEEE Comput. Soc. Press, 1994, pp. $566-568$.

[26] F. Murtagh, "A New Approach to Point-Pattern Matching," Publications of the Astronomical Society of the Pacific, vol. 104, no. 674, pp. 301307,1992

[27] X. Bai, X. Yang, L. Latecki, W. Liu, and Z. Tu, "Learning ContextSensitive Shape Similarity by Graph Transduction," IEEE Transactions on Pattern Analysis and Machine Intelligence, vol. 32, no. 5, pp. 861-874, 2010.

[28] B. Wang and Y. Gao, "Hierarchical string cuts: A translation, rotation, scale, and mirror invariant descriptor for fast shape retrieval," IEEE Transactions on Image Processing, vol. 23, no. 9, pp. 4101-4111, 2014.

[29] Y. Jiang, G. Kuang, W. Wang, L. Zhao, and B. Xiong, "Contour Matching Using the Affine-invariant Support Point Set," IET Computer Vision, vol. 8, no. 1, pp. 35-44, 2014.

[30] J. Canny, "A Computational Approach to Edge Detection," IEEE Transactions on Pattern Analysis and Machine Intelligence, vol. 8, no. 6, pp. 679-698, 1986.

[31] H. Ling and D. Jacobs, "Shape Classification Using the InnerDistance," IEEE Transactions on Pattern Analysis and Machine Intelligence, vol. 29, no. 2, pp. 286-299, 2007.

[32] J. Wang, X. Bai, X. You, W. Liu, and L. J. Latecki, "Shape Matching and Classification Using Height Functions," Pattern Recognition Letters, vol. 33, no. 2, pp. 134-143, 2012. 